\section{Fragen an}

\section{Prof. Dr. Ines Himmelsbach \\ Trägerin des Förderpreises der Wilhelm Woort-Stiftung}

\section{FORSCHUNGSSCHWERPUNKTE}

- Gerontologische Grundlagen

- Bildung, Wohnen und Gesundheit im Alter

- Altern mit Kompetenzeinbußen, insbesondere Sehbeeinträchtigung im Alter

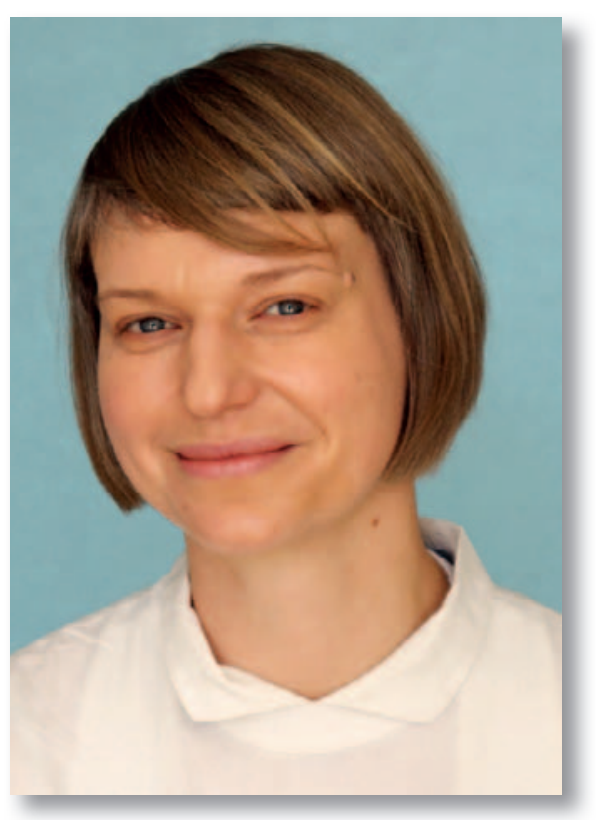

\section{BERUFLICHER WERDEGANG}

- Leitung des Instituts für Angewandte Forschung, Entwicklung und Weiterbildung (IAF)

- Seit 2014 Professorin für Soziale Gerontologie an der Katholischen Hochschule Freiburg im Breisgau

- Promotion im Fachbereich Erziehungswissenschaft an der Goethe-Universität Frankfurt am Main

- Studium an der Ruprecht-KarlsUniversität Heidelberg und der GoetheUniversität Frankfurt am Main (Erziehungswissenschaft und Romanistik)
Wie entstand die Idee für die «Beratungslandkarte bei Sehverlust im Alter»?

Die Idee entstand an der Auftaktveranstaltung des Aktionsbündnisses «Sehen im Alter», einem Aktionsbündnis initiiert vom Deutschen Blinden- und Sehbehindertenverband e.V. (DBSV) und von der Bundesarbeitsgemeinschaft der Senioren-Organisationen (BAGSO). In der dort entstandenen Arbeitsgruppe «Beraten und Begleiten» wurde schnell klar, dass sowohl für ältere Menschen selbst als auch für deren Berater ein besserer Überblick über die Beratungslandschaft gewährleistet werden müsse.

Was ist das Ziel dieses Forschungsprojektes?

Ziel ist eine erste wissenschaftliche Beschreibung und eine internetbasierte Landkarte von Beratungsangeboten für ältere Menschen mit Sehverlust in Deutschland.

Was hat Sie während der Arbeit daran oder nach Auswertung der Ergebnisse überrascht?

Überraschend waren für mich das hohe ehrenamtliche Engagement in diesem Bereich sowie die knappen finanziellen Ressourcen, unter welchen gute und wichtige soziale Beratungsarbeit geleistet wird.

Worin liegt die Relevanz Ihrer Ergebnisse für die praktische, klinische Arbeit eines Ophthalmologen?

Die Möglichkeit, sobald unsere Landkarte im Netz verfügbar ist, dass auch Ophthalmologen einfacher ihre Patienten an Beratungsangebote verweisen können.
Für welche Fachgruppen sind Ihre Ergebnisse darüber hinaus interessant?

Zunächst ist das Angebot für ältere Sehgeschädigte und ihre Angehörigen gedacht. Aber selbstverständlich sollen die Ergebnisse auch für die Altenhilfe und für alle Fachgruppen, die mit der Beratung älterer Menschen befasst sind, dienlich sein.

Der Preis ist mit $25000 €$ dotiert. Was machen Sie mit dem Preisgeld?

Es ist direkt in das Projekt eingeflossen und hat wesentlich die Stelle von Frau Anna Mielich, M.A., der wissenschaftlichen Mitarbeiterin des Projektes, finanziert. Weitere Förderer waren die Aktion Mensch und der DBSV.

Womit schaffen Sie für sich selbst und für Ihr Umfeld einen Ausgleich zu Ihrem Einsatz für die Forschung?

Gerne durch Wandern und Lesen und immer wieder durch gutes Essen.

\section{ANGABEN ZUM PREIS}

Für eine anwendungsnahe wissenschaftliche Begleitstudie zu einer modellhaften Initiative im Bereich der Altenversorgung vergibt die Wilhelm Woort-Stiftung alle 3 Jahre den mit $25000 €$ dotierten Förderpreis. Nachwuchswissenschaftler sowie Mediziner in außeruniversitären Einrichtungen, die an der Implementierung innovativer Instrumente oder Methoden arbeiten, können sich bewerben.

\section{KARGER}

Fax +497614520714 information@karger.com www.karger.com

\section{() 2016 S. Karger GmbH, Freiburg}

Prof. Dr. phil. Ines Himmelsbach

Katholische Hochschule Freiburg

Institut für Angewandte Forschung, Entwicklung und Weiterbildung

Karlstraße 63, 79104 Freiburg, Deutschland

ines.himmelsbach@kh-freiburg.de 